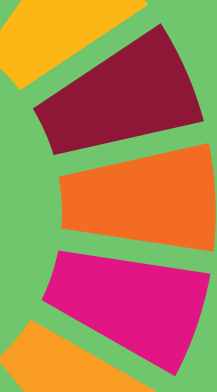

\title{
WORK4PROGRESS: PLATAFORMAS DE INNOVACIÓN PARA LA CREACIÓN DE EMPLEO
}

\section{WORKAPROGRESS: INNOVATION PLATFORMS FOR JOB CREATION}

\author{
Ariadna Bardolet \\ Fundación Bancaria "la Caixa" \\ abardolet@fundaciolacaixa.org \\ Marta Solsona \\ Fundación Bancaria "la Caixa" \\ msolsona@fundaciolacaixa.org
}

Fecha recepción artículo: 07/01/2020 • Fecha aprobación del artículo: 17/02/2020

\section{RESUMEN}

Los paradigmas de la cooperación internacional están cambiando y Work4Progress es un ejemplo de ello. El origen y objetivo de este programa, lanzado en 2017 por la Fundación "la Caixa" tras más de 20 años de experiencia en cooperación internacional, permite poner las herramientas de innovación social más avanzadas a disposición de los programas de cooperación internacional mediante plataformas de innovación abierta. Todo ello con el objetivo de crear empleo para mujeres y los jóvenes en situación de vulnerabilidad en India, Mozambique y Perú.

La principal novedad de Work4Progress es la incorporación de nuevas herramientas y metodologías en: 1) escucha e identificación de necesidades comunitarias, 2) co-creación y prototipado de nuevas soluciones, 3) exploración de instrumentos de escalado, 4) gobernanza, 5) sistemas de evaluación evolutiva y 6) estrategias de financiación.

Estas herramientas aplicadas a entornos complejos como los que trabaja Work4Progress contribuyen a incrementar el impacto de los programas, evitando propuestas "top-down" y generando nuevos modelos de colaboración.

Palabras clave: Innovación, Plataformas multiactor, Creación de empleo, Mujeres, Desarrollo sostenible. 


\section{ABSTRACT}

It is clear that the paradigms of international cooperation are changing, and Work4Progress is an example of this. The origins and goal of this programme, launched in 2017 by the "la Caixa" Foundation, provides the most advanced social innovation tools for international cooperation programmes via open innovation platforms. All this seeks to create employment for women and youngsters in a situation of vulnerability in India, Mozambique and Peru.

Its main innovation is the inclusion of new tools and methodologies in: 1) listening to and identifying community needs, 2) joint creation and prototyping of new solutions, 3) exploration of scaling instruments, 4) governance, 5) evolutionary evaluation systems, and 6) funding strategies.

When applied in complex environments such as the ones with which Work4Progress works, this tools help increase the impact of the programmes, avoiding top-down proposals and generating new models for collaboration.

Keywords: Innovation, Multi-stakeholder platforms, Job creation, Women, Sustainable development.

Ariadna Bardolet es Directora del Departamento de Programas Internacionales.

Se incorporó a la Fundación "la Caixa" en 1997, trabajando inicialmente en CosmoCaixa, el Museo de la Ciencia de la Fundación "la Caixa" en Barcelona.

En junio de 2000 comenzó a trabajar en el Programa de Cooperación Internacional y en febrero de 2009 asumió el cargo de Subdirectora del Área Internacional, actualmente Directora de Programas Internacionales, a cargo de temas de salud, educación, creación de empleo, acción humanitaria y desarrollo a nivel mundial en más de treinta países de los más vulnerables del mundo en los que la institución ha promovido diversos proyectos.

Ariadna Bardolet se licenció en Derecho por la Universidad de Barcelona así como en Dirección de Empresas en la Universidad ESERP de Barcelona, y cuenta con un Master en Globalización, Desarrollo y Cooperación de la Universidad de Barcelona y un PDD por IESE.

Marta Solsona es Responsable de Programas en Fundación "la Caixa". Actualmente está a cargo del programa internacional Work4Progress, cuyo objetivo es promover soluciones innovadoras para el empleo en India, Mozambique y Perú, promoviendo la transición de proyectos aislados a plataformas de innovación social. También es responsable del Programa de Desarrollo Socioeconómico, dirigido a generar oportunidades económicas para los más vulnerables en países de América Latina, África y Asia. Anteriormente trabajó en CosmoCaixa, el Museo de la Ciencia de Barcelona, donde estuvo a cargo de las exposiciones y actividades de divulgación científica.

Tiene un doctorado en geología (Universidad de Barcelona) y un posgrado en gestión de ONG e innovación social (ESADE-Instituto de Innovación Social). 
Los avances logrados en la reducción de la pobreza han sido notables en las últimas décadas. No obstante, según datos de la Organización Internacional del Trabajo, todavía hoy 172 millones de personas en el mundo siguen desempleados, afectando principalmente a mujeres y jóvenes. Al ritmo actual de progreso, será difícil alcanzar el Objetivo para el Desarrollo Sostenible número 8 fijado por Naciones Unidas cuyo reto es lograr un trabajo decente para todos. Es por ello que en los próximos años se requerirán todos los esfuerzos posibles, tanto por parte de actores públicos como privados, para lograr que podamos avanzar en la consecución de la Agenda 2030.

Este es el origen y objetivo del programa Work4Progress, lanzado en 2017 por el área internacional de la Fundación "la Caixa", que permite poner las herramientas de innovación social más avanzadas a disposición de los programas de cooperación internacional mediante plataformas multi-actor. Todo ello con la misión de lograr un empleo de calidad para jóvenes y mujeres vulnerables en India, Perú y Mozambique.

Este artículo presenta la metodología propuesta por el programa Work4Progress, su implementación en India, Mozambique y Perú, los principales resultados que se han obtenido hasta el momento, así como el esfuerzo que realiza Work4Progress para capturar y sistematizar todo el conocimiento generado con el fin de contribuir a una cooperación al desarrollo más innovadora y efectiva.

\section{LA FUNDACIÓN "LA CAIXA"}

Desde hace más de 110 años, la Fundación "la Caixa" está fuertemente comprometida con el progreso de las personas, así como en la construcción de una sociedad mejor para todos.

La entidad cuenta con una larga historia de defensa de las causas sociales, abordando algunas de las necesidades más urgentes en los campos de la acción social, educación, cultura y ciencia e investigación, tratando siempre de mantener una visión global y de innovar en todas sus acciones.

Cada año se impulsan más de 40.000 actividades y más de 11 millones de personas participan y se benefician del trabajo promovido por nuestra entidad, la mayoría en España, pero también, desde hace más de 20 años, en países de América Latina, África y Asia. El principal objetivo estratégico internacional es proporcionar oportunidades y combatir las desigualdades a través de programas que contribuyen al cumplimiento de los Objetivos de Desarrollo Sostenible de las Naciones Unidas.

Para ello las 4 líneas de acción en el ámbito internacional son:

- Salud global, especialmente centrados en la lucha contra las principales causas de mortalidad en países en vías de desarrollo, como la malaria y la neumonía.

- Educación, proporcionando acceso a la educación y formación de niños y jóvenes, especialmente mediante nuevas metodologías y tecnologías.

- Emergencias y acción humanitaria, mejorando las condiciones de vida de los refugiados, y especialmente de los niños que sufren desnutrición.

- Y la creación de empleo, mediante el programa Work4Progress, que fomenta el empleo de mujeres y jóvenes vulnerables y de movilización de las organizaciones de la sociedad civil.

Todos estos programas son implementados en colaboración con entidades locales, nacionales o internacionales con un largo recorrido en la cooperación internacional. Ello permite compartir sinergias y aprender unos de otros. 


\section{WORK4PROGRESS}

El programa Work4Progress responde a la necesidad de repensar la forma de promover los proyectos de cooperación internacional para el desarrollo, experimentar con nuevas herramientas para reforzar su impacto e incorporar un nuevo enfoque de innovación social.

Work4Progress se construye sobre la experiencia y el trabajo llevado a cabo por la Fundación "la Caixa" en cooperación internacional, desde su origen en 1997. Una larga trayectoria que ha permitido identificar las fortalezas y debilidades de las tradicionales convocatorias de apoyo a proyectos de desarrollo y proyectar la nueva estrategia de cooperación internacional hacia el futuro.

Las tradicionales convocatorias seleccionaban los proyectos de desarrollo socioeconómico más pertinentes a impulsar juntamente con entidades españolas y locales para la mejora de las condiciones de vida de la población más vulnerable en países de África, América Latina y Asia. Estas convocatorias gozaban de cierto reconocimiento por su regularidad, la aportación económica, así como por su especialización, principalmente basadas en desarrollo socioeconómico.

No obstante, los proyectos seleccionados en ocasiones eran geográfica y sectorialmente dispersos, y mayoritariamente, con distintas teorías de cambio intrínsecas.

Con el lanzamiento hace más de 2 años del programa Work4Progress se ofrece un nuevo enfoque para el desarrollo promoviendo innovación y el empleo de calidad para mujeres y jóvenes vulnerables mediante plataformas de innovación abierta focalizadas geográfica y sectorialmente.

El programa pasa de pensar en clave de proyectos aislados a ser una verdadera plataforma de innovación que conecta actores y acciones de forma más eficiente, con un específico enfoque en los resultados y la sostenibilidad a medio y largo plazo.

La principal novedad del mismo es la incorporación de nuevas herramientas y metodologías en: 1) escucha e identificación de necesidades comunitarias, 2) co-creación y prototipado de nuevas soluciones, 3) exploración de instrumentos de escalado, 4) gobernanza, 5) sistemas de evaluación evolutiva y 6) estrategias de financiación (figura 1). Todo ello mediante la concentración de esfuerzos en acciones transformadoras desde un punto de vista territorial, pero sobre todo metodológico.

Con Work4Progress se promueve la innovación en los productos, servicios y/o procesos, generando una nueva cultura de colaboración entre las entidades que están trabajando en una misma zona geográfica. La finalidad es incrementar su impacto y sostenibilidad, incorporando nuevos procesos de monitorización y evaluación constante que permiten adaptar el programa en tiempo real a las necesidades de cada lugar.

Tal y como menciona Gorka Espiau, director científico del programa, "esta forma de trabajar maximiza los recursos existentes, reduce el riesgo de la inversión y genera una estrategia de salida a medio y largo plazo para sus participantes. El elemento diferencial de una plataforma de estas características es que construye nuevas herramientas de escucha comunitaria, co-diseña las intervenciones junto a la ciudadanía y plantea la creación de una metodología colaborativa en lugar de proyectos aislados con la aspiración de construir un verdadero ecosistema de innovación en el espacio donde opera" (Espiau, G. 2018, pg. 7).

El programa cuenta actualmente con la implicación de una amplia red de organizaciones de la sociedad civil locales, sector privado, administraciones públicas, universidades y ONGD españolas. Este grupo de 
organizaciones forman las plataformas multi-actor de forma abierta dado que permiten incorporar en cualquier momento otros agentes públicos y/o privados en alguna o todas las fases del proceso.

El objetivo principal y final de Work4Progress es la creación de empleo. Tras más de dos años de implementación el programa ha conseguido llegar a más de 26.000 personas, diseñando y testando 75 prototipos de negocios que han contribuido a crear hasta el momento 559 microempresas, y con ello 1.609 nuevos empleos en India, Mozambique y Perú.

Work4Progress ha sido diseñado con una mentalidad de Do Tank pero también de Think tank. Es por este motivo que expertos nacionales e internacionales contribuyen a capturar todo el conocimiento que se va generando para compartirlo con centros de pensamiento y acción a nivel internacional.

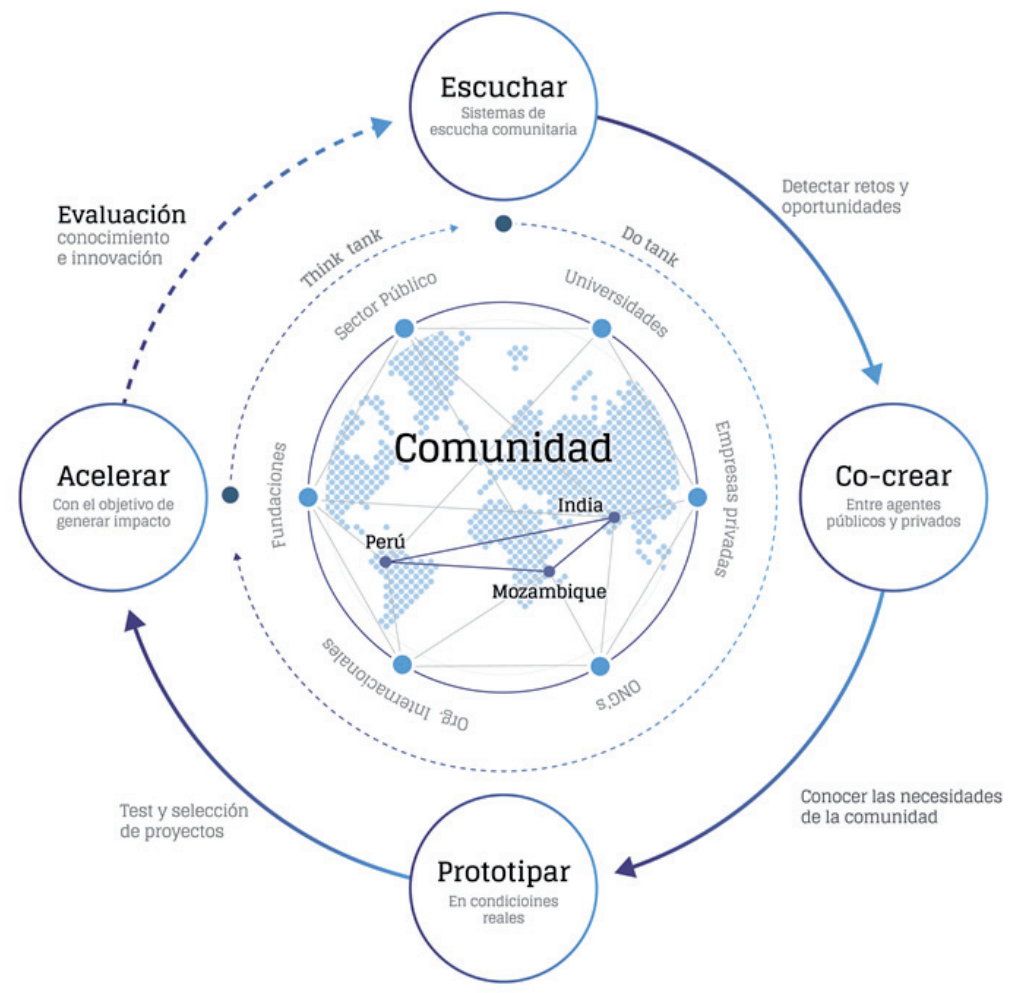

Figura 1.: Metodología del programa Work4Progress

Desde su creación Work4Progress incorpora estrategias de innovación en todos sus componentes de forma integral. Para ello promueve nuevas herramientas de escucha e identificación de necesidades en las comunidades, la co-creación y prototipado de nuevas soluciones junto a los socios y beneficiarios que potencien la promoción de empleo y la implementación de instrumentos que permitan el escalado de estos prototipos. 


\section{METODOLOGÍA DE IMPLEMENTACIÓN DE WORK4PROGRESS}

Work4Progress presta una atención especial al contexto organizativo y a los procesos de relación con todos los actores implicados. Para ello mantiene un modelo de gobernanza en el que participan las plataformas de organizaciones, sin que exista para ello un único liderazgo. Se busca generar una nueva cultura de colaboración entre las entidades que trabajan en una misma zona geográfica para que entre todas, se contribuya a incrementar la sostenibilidad a la acción.

El programa cuenta actualmente con la implicación de 35 organizaciones de la sociedad civil locales, sector privado, Administración, universidades y ONGD españolas.

Los equipos profesionales que impulsan el mismo son multi-institucionales y multi-disciplinarios, con perfiles de ciencias sociales, antropología, economía, ingeniería, y con conocimiento del territorio y del idioma local de las zonas de actuación.

En el marco de los consorcios de entidades en cada uno de los 3 países existe la figura del responsable del programa y líder de la plataforma, un responsable del proceso de evaluación, así como una persona encargada de la comunicación. Cada plataforma de entidades se reúne periódicamente para analizar la evolución y progreso de las acciones y reorientar actividades si es necesario. Se requiere de una coordinación y dinamización a nivel global que se reúne periódicamente y permanece interconectada.

Work4Progress permite en este caso a la Fundación "la Caixa" actuar como un socio facilitador a lo largo del proceso, aportando financiación, prestando apoyo técnico y formativo a las plataformas y atrayendo nuevos actores. Todo ello mediante personal experto tanto interno como externo a la entidad que da apoyo en todas las etapas del proceso desarrollando acciones de formación, intercambio y escucha activa al servicio de todas las organizaciones participantes, para corregir o a incorporar nuevas ideas en cualquier momento que se requiera.

Un comité formado por expertos aporta valor científico y social al programa en temas de cooperación internacional, innovación social y monitoreo y evaluación.

\section{LA FASE DE ESCUCHA Y CO-CREACIÓN EN WORK4PROGRESS}

Una de las principales novedades que incorpora el programa es la fase de escucha comunitaria. Tanto en el inicio como durante toda su implementación, la escucha comunitaria y la captación de narrativas son claves para comprender el sistema de valores, necesidades y aspiraciones de las comunidades.

Así lo afirma Jayne Engle, Directora del programa Cities for People en la Fundación McConnell "La escucha constituye la base de la participación y el diálogo, así como de la conciencia crítica y el cambio social. Por todo ello, la escucha colectiva y la búsqueda de sentido resultan fundamentales para la creación de plataformas de innovación orientadas a la generación conjunta de un cambio social" (Engle, J. y Slade S., 2018, pg. 23).

En el caso de Work4Progress, las plataformas en los tres países han llevado a cabo desde el inicio del programa acciones de escucha mediante entrevistas en profundidad, grupos focales, historias de vida, observación participativa, fotografía social, teatro de calle, desarrollo de perfiles etnográficos y sesiones de contraste con actores externos, que han permitido tener un diagnóstico muy detallado de las distintas regiones donde se implementa el programa. 
Todo ello ha permitido contar con datos económicos, indicadores sociales, de calidad de vida y de identidad cultural, expectativas de las poblaciones, así como mapas de retos y oportunidades para la generación de empleo de las regiones de los tres países en los que se actúa.

La escucha permanece activa durante todo el desarrollo del programa y en la fase de co-creación se involucra además de las comunidades, a otros actores locales y stakeholders, fomentando de este modo la cultura colaborativa.

\section{LA FASE DE PROTOTIPADO EN WORK4PROGRESS}

Durante la fase de prototipado, cada una de las 3 plataformas identifica las diferentes líneas de negocio y posibles iniciativas empresariales con el objetivo de validar su viabilidad, reforzar aquellas que generen empleo de modo más eficiente y valorar su replicabilidad y/o escalabilidad en las zonas de intervención del proyecto. Aquellos prototipos que estén más maduros y conectados con la comunidad podrán pasar en un futuro a una fase de aceleración.

Dentro de los prototipos elegidos se incluyen iniciativas de pequeña escala o comunitarias (iniciativas basadas en la comunidad), empresas de tamaño medio que involucran a organizaciones ya existentes en el territorio, y actuaciones a gran escala que implican a las instituciones locales, ONGD y empresas privadas.

Hasta el momento con el programa Work4Progress se han promovido 75 prototipos de microempresas, servicios y productos de apoyo al emprendedor vinculados a la transformación de productos agrícolas (hongos, alpaca, queso, miel), microempresas de transporte, cybercafés, centros de desarrollo empresarial, etc. en India, Mozambique y Perú.

\section{WORK4PROGRESS EN PERÚ}

En el caso del Perú el programa se implementa en dos regiones: la provincia de Quispicanchi, región de Cusco, en el sur andino del país, y la provincia de Condorcanqui, región de Amazonas, en el norte peruano.

Ambas regiones son distintas en su entorno geográfico y en su dinámica territorial, pero cuentan con elementos comunes: su población es en su gran mayoría indígena (quechua en Cuzco y awajún - wampis en Amazonas), y pertenece a sectores altamente vulnerables, con potencial para el desarrollo de sus economías.

La región del Cusco, específicamente la provincia de Quispicanchi, es una población más articulada con el mercado local. Por este motivo Work4Progress Perú ha iniciado su implementación en esta región, con el objetivo de entender cómo se promueve el trabajo con jóvenes y mujeres en la zona para exportarlo posteriormente a una zona menos articulada al mercado, como es la de Condorcanqui.

Los beneficiarios de la intervención son jóvenes y mujeres en su mayoría indígenas, considerados por los indicadores nacionales como familias vulnerables en términos de pobreza. Su principal actividad económica es la agricultura y el comercio a pequeña escala. Esta población joven de entre 15 a 29 años representa el 
$25 \%$ total de la población del departamento de Amazonas y el 27\% para el departamento de Cusco, con una incidencia de la pobreza del $47 \%$ y $14 \%$ para Amazonas y Cuso respectivamente (Instituto Nacional de Estadística e Informática - INEI, 2018).

La plataforma de entidades a cargo de la ejecución del Programa en el Perú se inició en 2018 con 6 socios: Entreculturas como ONG española y líder de la plataforma, Fe y Alegría Perú, presente en ambos territorios de intervención y coordinando la evaluación, comunicación y los equipos territoriales; CCAIJO, socio líder en temas productivos en Quispicanchi; SAIPE, socio líder en temas productivos en Condorcanqui; AVSI, entidad referente en temas de empleabilidad juvenil y cadenas de valores, y la Universidad Antonio Ruiz de Montoya, apoyando tanto a los procesos de investigación y producción de conocimiento como a la promoción de prototipos tecnológicos. Además, el Instituto GRADE está implementando una evaluación experimental en la región de Quispicanchi. A partir de enero de 2020 una nueva red de entidades liderada por Codespa y con presencia de la Universidad de San Marcos, ha entrado a formar parte de la plataforma de Perú, con el objetivo de complementar y apoyar la red inicial en la fase de aceleración de las actividades generadoras de empleo.

Tras una fase inicial de 6 meses para el diagnóstico y co-creación con casi 1.500 participantes de 14 comunidades distintas (10 de Quispicanchi y 4 de Condorcanqui) el consorcio de entidades ha definido e iniciado la implementación de 17 prototipos. De todo ellos, 5 corresponden a iniciativas de apoyo al desarrollo empresarial e inserción laboral ( 2 centros de desarrollo empresarial, una bolsa de trabajo para jóvenes, circuitos formativos en habilidades blandas para mejorar la empleabilidad de jóvenes de educación secundaria y para el liderazgo productivo de emprendedoras) y 12 a micro-empresas (secado de hongos de pino, fibra de alpaca, producción de quesillo, purificación de agua con energía solar, etc.).

Según Mimi Cuq, responsable del programa Work4Progress en Perú: "La implementación de un programa como Work4Progress propone una nueva metodología, una mirada más atenta y abierta a la complejidad de la realidad, y nos desafía también a proponer nuevas ideas, a arriesgar, a atreverse para mejorar, mejorar nuestra comprensión de la realidad, mejorar nuestras capacidades de trabajar con otros, mejorar los resultados de nuestras intervenciones para contribuir a la mejora de la calidad de vida de la población."

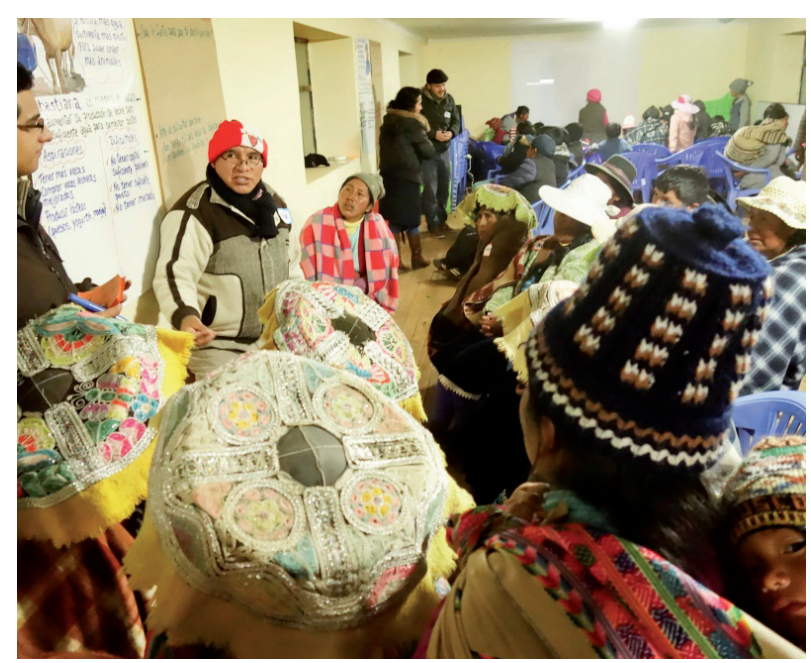

Foto 1: Taller de devolución de la fase de escucha a las comunidades (Quispicanchi, Cusco, 2018).

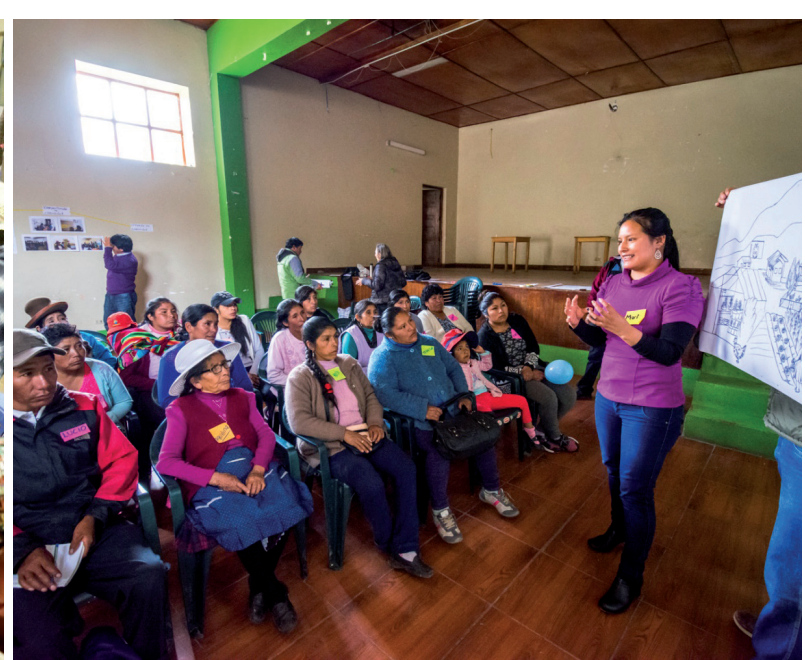

Foto 2: Taller de co-creación en Quispicanchi, Cusco, 2018 


\section{WORK4PROGRESS EN INDIA}

En India, dos tercios de la población y el $70 \%$ de su fuerza laboral viven en áreas rurales, lo que significa el $46 \%$ del ingreso nacional (Centre for Monitoring the Indian Economy, 2018). Las proyecciones de NITI Aayog indican que para 2050, la mitad de la población en India seguirá siendo rural (Chand, R., Srivastava, S. K. and Singh, J., 2017, pg. 1). Según el Centro de Monitoreo de la Economía de la India, más del $90 \%$ de la fuerza laboral de la India está en trabajos informales (Centre for Monitoring the Indian Economy, 2018).

En las zonas rurales de India existe una necesidad de promover el crecimiento económico y la creación de empleo, con el fin de proporcionar niveles de vida dignos. La falta de capacitación, acceso a información y a servicios relevantes, así como la baja innovación, conllevan serias dificultades para seguir promoviendo el espíritu empresarial en el país.

En este sentido, las microempresas juegan un papel fundamental para abordar estos desafíos complejos, contribuyendo a crear empleo local.

Work4Progress en India contribuye a la mejora del ecosistema rural al promover las inquietudes comerciales de las mujeres y los jóvenes en las comunidades rurales, crear empresas ejemplares sostenibles y escalar aquellas que tienen más éxito.

Desde 2017, la organización india Development Alternatives lidera la plataforma en el país junto a una red de socios con diversos grados de participación, incluidas organizaciones de la sociedad civil (Manaviya Dristikon Sewa Samiti, Swami Vivekanand Sewa Sansthan), universidades (Universidad Bundelkhand), federaciones de agricultores (Organización de Productores Agricultores de Sakshi), agregadores del mercado (Crop Connect), programas gubernamentales (Banco Nacional de Agricultura y Desarrollo Rural, Misión Nacional de Medios de Vida Rurales), y empresas privadas (Quest Alliance). Recientemente se han incorporado nuevos aliados incluyendo las organizaciones Alianza por la Solidaridad y Action Aid India.

El programa se desarrolla en 60 comunidades en las regiones de Bundeklhand (distritos de Jhansi y Niwadi) y del este de Uttar Pradesh (distritos de Mirzapur y Bhadohi).

En el período de escucha, participaron más de 3.500 jóvenes y mujeres y en la fase de co-creación hubo más de 2.500 participantes en grupos focales y capacitaciones; también se realizaron entrevistas estructuradas a más de 600 empresarios. Se organizaron los concursos "Kaun Banega Business Leader" para captar ideas de negocios en las aldeas (de las cuales se recibieron 1.000 solicitudes) y se organizaron varios eventos regionales sobre creación de empleo.

Como resultado de todo esto, se han puesto en marcha 46 prototipos de iniciativas generadoras de empleo que han llevado a la implementación de 527 microempresas, generando 1.122 empleos indirectos y directos hasta el momento. Del total de prototipos, 15 de ellos son de apoyo y fomento del ecosistema emprendedor, como los espacios seguros para mujeres, las ferias empresariales, la red de quioscos de información para emprendimientos liderados por jóvenes y las coaliciones de actores regionales. Los 31 restantes son prototipos de microempresas, entre las que se encuentran plantas comunitarias de suministro de agua, empresas ecológicas del sector agrícola, una fábrica de helados, una empresa de vermicompost, y empresas de transporte ecológicas.

Uno de los prototipos más exitosos es el de micro-empresas de triciclos rickshaw, un vehículo muy 
popular en India, alimentados por energía eléctrica. Estos rickshaw son conducidos por mujeres que ofrecen el servicio a otras mujeres y niñas. Los rickshaw eléctricos son más silenciosos, ecológicos y económicos de mantener que los que utilizan combustibles fósiles, y menos agotadores que los rickshaw tradicionales impulsados con pedales. Hasta la fecha se han implantado 3 microempresas de rickshaw en las dos regiones donde opera Work4Progress, y se está trabajando en un modelo para escalarlas a otras comunidades.

El programa Work4Progress en India también ha establecido 2 coaliciones regionales, formadas por representantes de organizaciones de la sociedad civil, instituciones microfinancieras (IMF), agencias del gobierno local, proveedores de tecnología, instituciones académicas y empresarios. Estas coaliciones actúan como "lobby" a favor de los microempresarios.

A través de las coaliciones regionales, se ha establecido acuerdos de colaboración con 6 entidades microfinancieras (banco HDFC, Sonata Micro Finance, Cashpor Micro Credit, SKS Microfinance, Bandhan Small Finance Bank y Unibase Micro Finance), para favorecer el acceso a productos financieros para los micro-emprendedores.

Según Kanika Verma, responsable de Work4Progress en India: "Work4Progress actualmente representa la innovación en el desarrollo de microempresas, un enfoque en el que se crea un ecosistema habilitador con las partes interesadas locales para construir una cultura de emprendimiento, acelerando el ritmo al que se generan empleos en las comunidades más vulnerables".

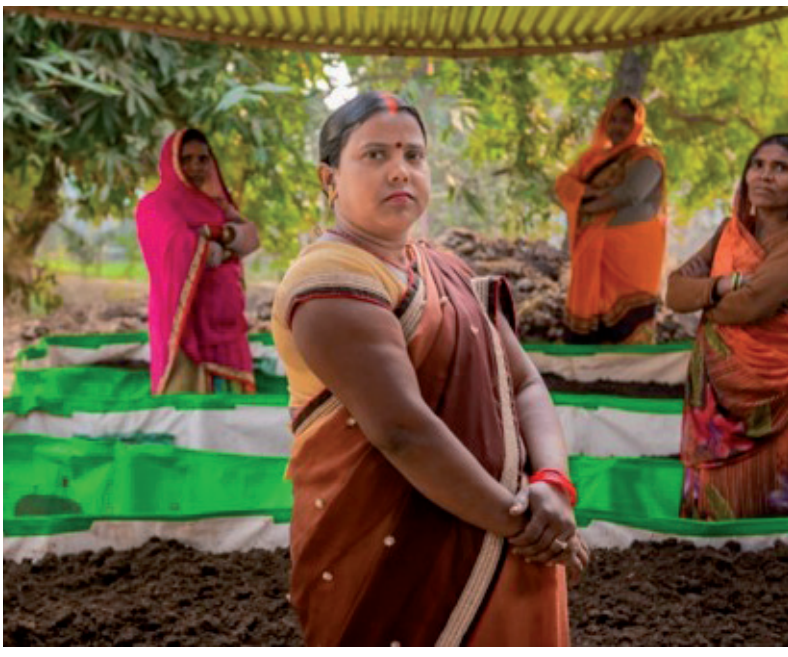

Foto3.AshaDevi,emprendedoradevermicompostaje en Mirzapur

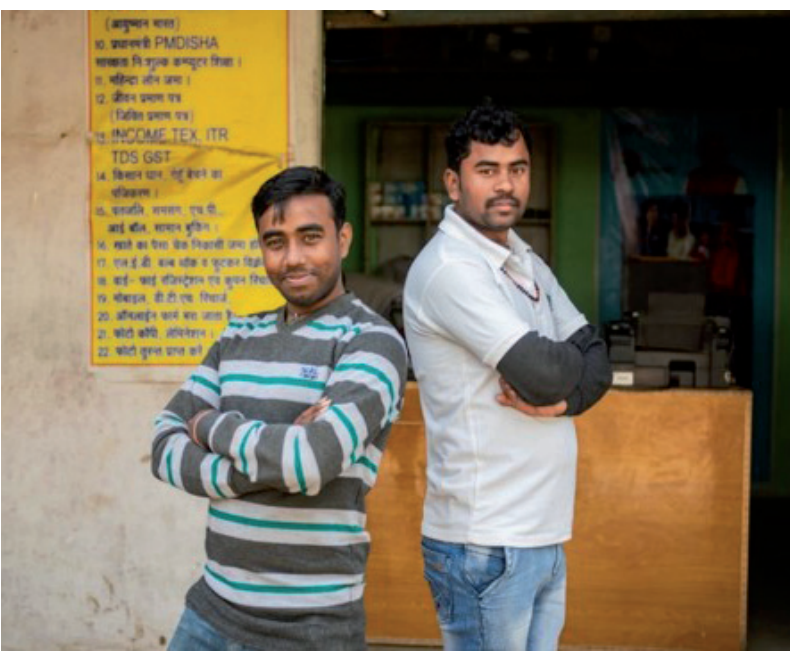

Foto 4. Gulshan Bind, quiosco de servicios para el emprendedor

Asha Devi (foto 3), una emprendedora de vermicompostaje en Mirzapur, estableció una empresa por primera vez en su vida con el apoyo del programa Work4progress. En los últimos cinco meses, sus ventas se han duplicado. Para satisfacer la mayor demanda de producto comercializa también productos de otros fabricantes de vermicompost y vende su abono empaquetado a mejores precios. Este emprendimiento ha creado una red de demanda y oferta entre empresarios. También se ha convertido en agente de cambio para que otras mujeres de mediana edad en su comunidad establezcan una fuente de ingresos independiente. 
Gulshan Bind (foto 4), diversificó su "quiosco de servicios para el emprendedor"1 agregando más información sobre opciones de empleo, lo que aumentó sus ingresos en un 30\%. "Quería quedarme en mi pueblo y dar información confiable sobre nuevas oportunidades a los jóvenes como yo" afirma Gulshan. Su empresa está situada en una de las áreas más concurridas de la aldea Lachhapatti en Mirzapur, y brinda servicios de apoyo empresarial y empleo a 1.500 residentes de 15 aldeas.

\section{WORK4PROGRESS EN MOZAMBIQUE}

En Mozambique, el programa se desarrolla principalmente en la provincia de Cabo Delgado, una provincia periférica en el norte de Mozambique afectada durante 2019 por el paso del ciclón Kenneth.

La población de Cabo Delgado asciende a 1,86 millones, con un 52\% de mujeres (Barrenechea, A. 2015, pg. 12). Los datos más recientes indican que la tasa de desempleo es del $25,5 \%$ para las mujeres (Barrenechea, A. 2015, pg. 15). El $90 \%$ de los empleos son en el sector de la agricultura a pesar de que la productividad es extremadamente baja (Barrenechea, A. 2015, pg. 15). Sólo el 6,4\% recibe un salario, mientras que el 63,1\% es autónomo y el 30,7\% trabaja sin remuneración alguna (Instituto Nacional de Estadística - INE, 2017, pg. 61). Los autónomos trabajan principalmente en micro y pequeñas empresas del sector informal. Sólo el 6,1\% de la mano de obra en Cabo Delgado trabaja en actividades no relacionadas con la agricultura (Instituto Nacional de Estadística - INE, 2017, pg. 61).

Las mujeres están expuestas a un mayor nivel de inseguridad y a la falta de protección social básica, además de estar socialmente atadas a la mayor parte de cargas del hogar y de los cuidados.

El empleo asalariado existe sólo en el $4 \%$ de los hogares, debido principalmente a la falta de inversiones en oportunidades de generación de empleo, limitadas intervenciones para el desarrollo de recursos humanos y falta de capacitación profesional (Instituto Nacional de Estadística - INE, 2017, pg. 61).

Casi el 88\% de la población económicamente activa de Cabo Delgado se dedica a la agricultura, la silvicultura, la pesca y las actividades extractivas, mientras que el $9 \%$ trabaja en el sector terciario y los restantes se dedican a actividades del sector secundario (Barrenechea, A. 2015, pg. 53). El acceso a los servicios financieros es disperso y básico y el acceso a los sistemas de seguros, en particular los sistemas vinculados con el clima (por ejemplo, la agricultura y la pesca), es prácticamente inexistente.

La plataforma Work4Progress en Mozambique está liderada por Ayuda en Acción y formada también por Mundukide, ESSOR, Espaço Livre Juventude (ELJ), Adel, Technoservey la Universidad Politécnica de Maputo. En la plataforma también participan empresas, bancos locales y los servicios distritales de la administración. Recientemente, se ha incorporado una nueva red de actores, liderada por Cesal y que incluye la universidad Unilurio, con el objetivo principal de ampliar la actuación del programa a Maputo

Durante la primera fase de escucha y diagnóstico en la región, entre las principales necesidades detectadas fueron la falta de acceso a crédito para los pequeños productores, la necesidad de articular un mercado en el que comercializar los productos, la necesidad de combustibles para los hogares más eficientes y respetuosos con el medioambiente, así como la necesidad de ofrecer formación para la inclusión laboral para jóvenes.

\footnotetext{
${ }^{1}$ Los quioscos de servicios para el micro-emprendedor son pequeñas empresas cuyo modelo de negocio se basa en ofrecer servicios a los emprendedores, desde conexión a internet y trámites burocráticos a información sobre programas del gobierno, ofertas de trabajo, etc.
} 
Durante el proceso de co-creación intervinieron todas las organizaciones que formaban parte de la plataforma Work4Progress en Mozambique, otros actores relevantes (gobiernos locales, empresas privadas de la zona, etc.) y la propia población local.

En la fase de prototipado se han puesto en marcha 12 prototipos que incluyen microempresas como las de energías renovables (cocinas mejoradas y carbón vegetal), de producción de miel con colmenas mejoradas, de productos hortícolas o distribución de huevos. Se han diseñado también prototipos de más alcance: la construcción de infraestructuras en la zona de Cabo Delgado para favorecer la comercialización justa en la región, 3 líneas de formación profesional que ha formado a 200 jóvenes de la zona, un fondo de garantía para facilitar el acceso a crédito a los pequeños productores y la creación de una asociación "AMO-HORTA" para representar a los productores de vegetales y frutas de la región, fomentar el consumo de productos locales y sobre todo incidir en el gobierno y consumidores para crear políticas de apoyo al sector.

Tal y como destaca Jaime Díaz, coordinador de Work4Progress en Mozambique: "La implementación de algunos de estos prototipos se ha beneficiado de nuevas alianzas puesto que ha permitido la entrada de nuevas entidades colaboradoras a la Plataforma como las empresas Elaco Orrera y Olima Agronegocio, el Banco Comercial e de Investimento $\mathrm{BCl}$ y los servicios distritales de la administración. En el caso de $\mathrm{BCl}$ se inició un acuerdo en marzo 2019 para sentar las bases técnicas del denominado Fondo de Garantía Work4Progress que actúa como palanca para que los pequeños productores agrícolas de Cabo Delgado puedan producir en pequeña escala con precios justos, incrementando su renta y contribuyendo a la mejoría de su calidad de vida y empleabilidad así como a la sostenibilidad de sus negocios. Con un valor total de 100.000 euros y un valor máximo del préstamo a cada agricultor seleccionado de 2.200 euros la finalidad es dar apoyo a la campaña de producción agrícola de los beneficiarios del programa Work4Progress."

\section{WORK4PROGRESS COMO THINK TANK}

Además de su trabajo en el terreno, Work4Progress promueve espacios de generación de conocimiento para la incorporación de metodologías de innovación social en el campo de la cooperación internacional para el desarrollo. El programa se ofrece como "living lab" para testar nuevas metodologías que puedan ser de utilidad para otras instituciones filantrópicas, gobiernos o entidades especializadas en el desarrollo internacional.

Una narración adecuada del proceso por parte de las entidades implicadas, puedecontribuir al fortalecimiento del proceso formativo, al seguimiento y evaluación de resultados del programa Work4Progress, y al traspaso de la metodología a otros actores sociales.

Para ello, desde su lanzamiento y con el objetivo de compartir todo el know-how adquirido con el programa Work4Progress, se promueve la organización de:

\section{ACTOS DE DIVULGACIÓN Y CONTRASTE:}

Cada año se organizan en India, Mozambique y Perú, los 3 países de acción de Work4Progress, eventos, conferencias y talleres de debate y discusión sobre el trabajo en plataforma y las nuevas metodologías de cooperación dotando a los grupos de interés de un espacio de diálogo y escucha. Se aprovecha estos foros 
para presentar también los avances y evolución de la implementación del programa en cada uno de los países, tanto con los socios como con los distintos stakeholders implicados y posibles colaboradores futuros de la plataforma.

También anualmente se organizan en España encuentros con todos los miembros de las plataformas y representantes del sector con el objetivo de compartir las buenas prácticas y los avances en cada uno de los países donde se implementa Work4Progress, con el objetivo de establecer puntos de conexión y plantear estrategias de progreso.

Hasta el momento en total se han impulsado 12 eventos nacionales e internacionales que han contado con la participación de más de 1.000 profesionales de la cooperación internacional y la innovación social.

El programa ha sido reconocido por UNDP como modelo de buena práctica de trabajo en Plataformas de Innovación abierta (UNDP y "la Caixa" Foundation, 2019, pg. 15). Todo ello en el marco de un evento organizado en Nueva York en marzo de 2019 con los equipos de innovación de UNDP, para compartir la metodología del programa junto a representantes de los principales organismos internacionales. El Banco Mundial en 2019 también ha seleccionado Work4Progress para su portfolio "Solutions for Youth Employment", en el que incluyen 30 de las mejores iniciativas en creación de empleo juvenil a nivel mundial (Singh, S., 2020, pg. 51).

\section{PUBLICACIONES Y MATERIALES FORMATIVOS PARA ORGANIZACIONES NO GUBERNAMENTALES Y DE LA SOCIEDAD CIVIL:}

El enfoque del programa Work4Progress basado en metodologías de innovación social y la creación conjunta es relativamente nuevo y exige nuevas herramientas para la formación, el monitoreo, la evaluación y el aprendizaje en toda la cartera de actividad.

Las organizaciones de las plataformas poseen niveles de formación diferente, limitada disponibilidad temporal, así como barreras idiomáticas y de conectividad. Además, es importante facilitar el aprendizaje basado en la experiencia y avanzar en la sistematización del mismo para el futuro. Los resultados de la sistematización de este conocimiento debe quedar disponible y ser aprovechable por y para otras organizaciones que puedan unirse a las Plataformas actuales o futuras.

Es por este motivo que, junto al Centro de Innovación en Tecnología para el Desarrollo Humano de la Universidad Politécnica de Madrid, se ha desarrollado un programa de formación con el objetivo de incorporar metodologías de innovación social en el campo de la cooperación internacional.

El mismo pone al alcance de los socios de programa, pero también de las organizaciones no gubernamentales del sector y otras entidades interesadas, una serie de módulos on-line que promueven la innovación social en el ámbito del desarrollo incidiendo en las fases de escucha, co-creación, prototipado, evaluación y comunicación, y poniendo como ejemplos prácticos las actividades desarrolladas sobre el terreno en el marco de Work4Progress². Además, también se han elaborado 4 guías prácticas sobre la metodología de este programa ${ }^{3}$.

\footnotetext{
${ }^{2}$ https://obrasociallacaixa.org/es/internacional/empleo/aula-virtual-modulo-i-escucha/clase-1

${ }^{3}$ https://work4progress.org/new-practical-guides-about-listening\%2C-co-creation\%2C-prototyping-and-evaluation-775
} 


\section{WORK4PROGRESS VIRTUAL L@B, UNA COMUNIDAD SIN BARRERAS GEOGRÁFICAS:}

Work4Progress cuenta con una plataforma virtual ${ }^{4}$ que permite el monitoreo permanente de los resultados y la interconexión entre todos los socios en los tres países (foto 5).

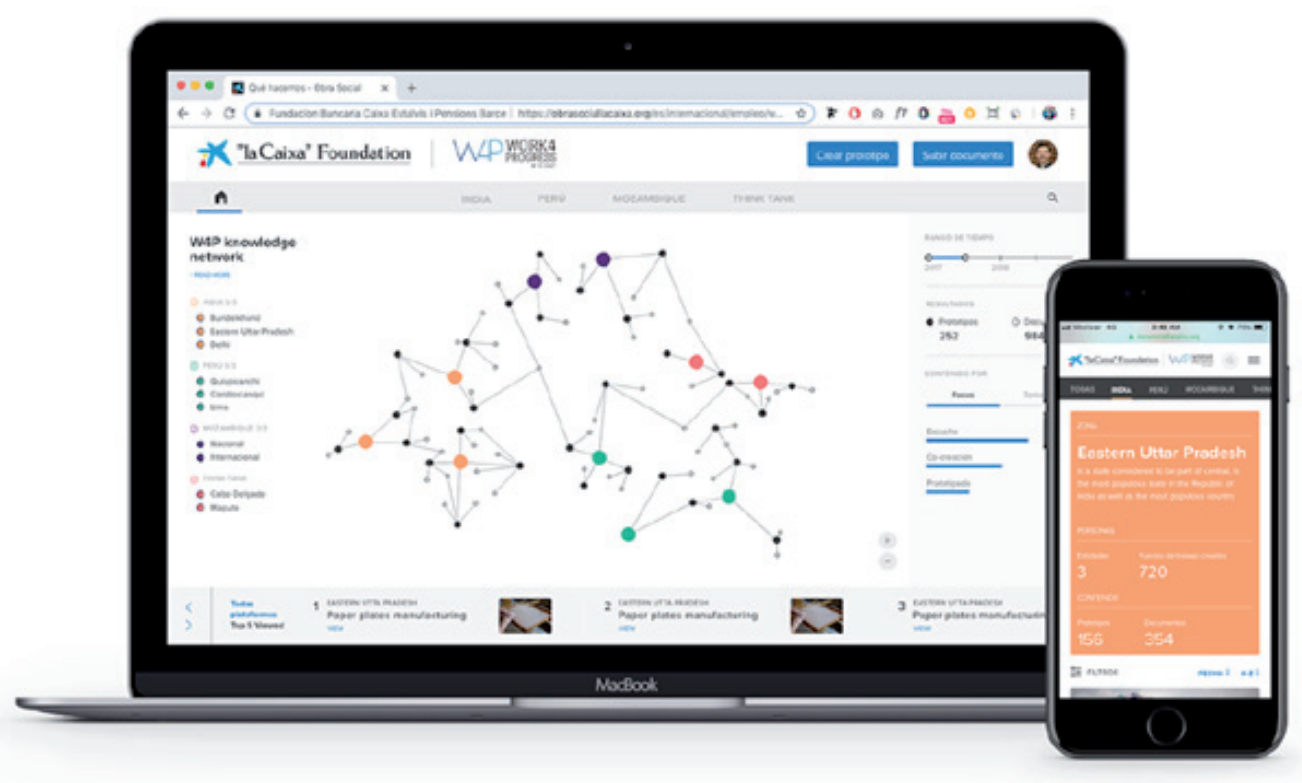

Foto 5: Work4Progress Virtual L@b

\section{CHALLENGE DE INNOVACIÓN TECNOLÓGICA:}

Inspirándose en los "Grand Challenges" o la idea de articular los desafíos globales para centrar los esfuerzos en dar respuesta a necesidades tecnológicas de las plataformas e introducir nuevos socios, se lanzó en 2019 un desafío de innovación abierto, destinado a apoyar el trabajo que se está llevando a cabo actualmente en los territorios de Work4Progress relacionados con la agricultura, la igualdad de género y la optimización de recursos naturales. Los proyectos seleccionados tienen como objetivo la creación de espacios de co-working virtuales para mujeres jóvenes que permitan promover el emprendimiento en India, la introducción de innovaciones tecnológicas para la mejora del uso del agua en procesos productivos agrícolas en Perú o la introducción de tecnologías agrícolas para mejorar la eficiencia de los cultivos en Mozambique. 


\section{EL MONITOREO Y EVALUACIÓN EN WORK4PROGRESS:}

La innovación social demanda una forma diferente de medir y evaluar los resultados de su impacto. Sin abandonar los parámetros cuantitativos que nos ayudan a presentar la evidencia del impacto, es necesario combinarlos con nuevos indicadores cualitativos. Junto a los mismos es necesario incorporar además equipos de evaluación evolutiva durante todo el proceso de forma que se puedan corregir errores y adaptar las iniciativas en tiempo real de cada comunidad para construir sus propias soluciones (Fisher, J. 2018, pg. 64).

Con este objetivo el Programa Work4Progress ha iniciado una macroevaluación para realizar un estudio comparativo, cualitativo y cuantitativo del mismo, diseñar y apoyar la implementación de una "evaluación evolutiva" y generar lecciones aprendidas y buenas prácticas en plataformas de innovación para el desarrollo.

El programa cuenta con una batería de indicadores de medición de impacto económico y social, definidos por los propios miembros de las plataformas, tales como número de prototipos/empresas/puestos de trabajo creados y fortalecidos o el cambio de narrativas vinculadas con la creación de empleo en la región.

Work4Progress recibe también la asistencia técnica del programa de voluntariado internacional de "la Caixa". Bajo el nombre de CooperantesCaixa, unos 15 empleados de la entidad viajan anualmente a los países de implementación del programa, para realizar asistencias técnicas en ámbitos como finanzas, estudios de mercado, estrategia, comunicación, etc.

\section{CONCLUSIONES}

La Agenda 2030 plantea importantes retos que hacen necesario identificar fórmulas y metodologías innovadoras para ser compartidas y que contribuyan a la mejora de la eficiencia e impacto de los programas de cooperación internacional.

Ante esta realidad las entidades del sector privado deben actuar como impulsoras de procesos de transformación, evitando focalizarse única y exclusivamente en la gestión y transferencia de recursos. En este marco de transformación además la experiencia ha demostrado que las alianzas y las redes siempre ofrecen resultados más eficaces que un conjunto de acciones puntuales. Es por todo ello que la colaboración y el aprendizaje compartido son ejes clave para el desarrollo socioeconómico de una región.

Siguiendo esta visión se concibe el programa Work4Progress lanzado por Fundación "la Caixa" en 2017 que promueve plataformas de innovación abierta, que busca las sinergias y la complementariedad de la labor de las organizaciones, conectando soluciones locales con problemas globales $y$, todo ello, con el fin de crear oportunidades laborales para mujeres y jóvenes en India, Mozambique y Perú.

En estos primeros años de implementación, el Programa ha adquirido una suma de aprendizajes que están contribuyendo a cambiar culturas organizativas y obtener importantes resultados para la creación de empleo. No obstante, la complejidad implícita en un programa de innovación social aplicado en contextos cambiantes como es el caso de Work4Progress presenta también importantes desafíos.

Es por ello que en estas circunstancias todavía se hace más necesario el foco de nuevas herramientas que aplicadas a entornos complejos como los que trabaja Work4Progress contribuyan a incrementar el impacto de los programas, evitando propuestas "top-down" y generando nuevos modelos de colaboración. Los paradigmas de la cooperación internacional están cambiando y Work4Progress es un buen ejemplo de ello. 


\section{REFERENCIAS BIBLIOGRÁFICAS}

- Anuario estadístico, 2017. Instituto Nacional de Estadística (INE), Moçambique.

- Barrenechea, A.: Situaçao atual das mulheres em Cabo Delgado. Action Aid Moçambique, 2015

- Chand, R., Srivastava, S. K. and Singh, J.: Changing Structure of Rural Economy of India Implications for Employment and Growth. National Institution for Transforming India (NITI Aayog), 2017

- Encuesta Nacional de Hogares sobre Condiciones de Vida y Pobreza, 2018. Instituto Nacional de Estadística e Informática (INEI), Perú.

- Engle, J y Slade S.: Escuchar para promover le cambio social: herramientas transformadoras que impulsan la innovación comunitaria, en: Plataformas que activan la innovación, Fundación Bancaria "la Caixa", 2018.

- Espiau, G.: Plataformas de innovación social para el desarrollo, en: Plataformas que activan la innovación, Fundación Bancaria "la Caixa", 2018.

- Fisher, J.: Consideraciones sobre el seguimiento, evaluación y aprendizaje en las plataformas de innovación social, en: Plataformas que activan la innovación, Fundación Bancaria "la Caixa", 2018.

- Open Innovation Platforms for Sustainable Development, V.V.A.A., UNDP y "la Caixa" Foundation, 2019.

- Singh, S.: S4YE'S Impact Portfolio. A factbook. Solutions for Yputh Employment, World Bank. Enero, 2020.

- Unemployment in India, Centre for Monitoring the Indian Economy, August 2018. 